PSU/TH/193; hep-ph/9801384

\title{
SECOND ORDER IN MASS RATIO RADIATIVE-RECOIL CORRECTIONS TO HYPERFINE SPLITTING IN MUONIUM
}

\author{
Michael I. Eides * \\ Department of Physics, Pennsylvania State University, University Park, PA 16802, USA ${ }^{\dagger}$ \\ and Petersburg Nuclear Physics Institute, Gatchina, St.Petersburg 188350, Russia ${ }^{\ddagger}$ \\ Howard Grotch ${ }^{\S}$ \\ Department of Physics, Pennsylvania State University, University Park, PA 16802, USA \\ Valery A. Shelyuto ** \\ D. I. Mendeleev Institute of Metrology, St.Petersburg 198005, Russia
}

(January, 1998)

\begin{abstract}
Radiative-recoil corrections to hyperfine splitting in muonium of orders $\alpha(Z \alpha)(m / M)^{2} E_{F}$ and $\left(Z^{2} \alpha\right)(Z \alpha)(m / M)^{2} E_{F}$ are calculated. These corrections are of the second order in the small electron-muon mass ratio. An analytic expression $\left[\left(-6 \ln 2-\frac{3}{4}\right) \alpha(Z \alpha)-\frac{17}{12}\left(Z^{2} \alpha\right)(Z \alpha)\right]\left(\frac{m}{M}\right)^{2} E_{F}$ is obtained. Numerically the correction is equal to $-0.0351 \mathrm{kHz}$ and is of the same order of magnitude as the expected accuracy of the current Los Alamos experiment to measure the hyperfine splitting.
\end{abstract}

PACS numbers: 36.10.Dr, 12.20.Ds, 31.30.Jv, 32.10.Fn

Typeset using REVTEX

\footnotetext{
*E-mail address: eides@phys.psu.edu, eides@lnpi.spb.su

${ }^{\dagger}$ Temporary address.

$\ddagger$ Permanent address.

§E-mail address: h1g@psu.edu

**E-mail address: shelyuto@onti.vniim.spb.su
} 


\section{INTRODUCTION}

The hyperfine splitting in the ground state of muonium has been measured with high precision [1]

$$
\Delta \nu_{\mathrm{Mu}}(n=1)=4463302.88(16) \mathrm{kHz} \quad \delta=3.6 \cdot 10^{-8},
$$

and there are further opportunities for improving the accuracy. The lifetime of the higher hyperfine state is extremely large $\tau=1 \cdot 10^{13} \mathrm{~s}$ and gives negligible contribution to the linewidth. Thus the linewidth is completely defined by the muon lifetime $\tau_{\mu} \approx 2.2 \cdot 10^{-6} \mathrm{~S}$ which leads to the width $\Gamma_{\mu} / h=72.3 \mathrm{kHz}$. The experimental accuracy attained thus far corresponds to measuring the energy splitting with an experimental error of $\Delta \nu_{\text {exp }} /\left(\Gamma_{\mu} / h\right) \approx$ $2.2 \cdot 10^{-3}$ of the natural linewidth. A new experiment is now running at Los Alamos [2], with the goal of measuring $\Delta \nu_{\mathrm{Mu}}(n=1)$ with a precision of about $1 \cdot 10^{-8}$, or at the level of a few hundredths of a kilohertz.

In anticipation of the long-awaited results from this precision experiment, recent years have witnessed a surge of theoretical activities in this field. A tedious calculation of the purely radiative corrections of order $\alpha^{2}(Z \alpha) E_{F}$ [3] 9 ] was followed by works [10,7],11, 12] where leading corrections of higher orders $\left(\alpha(Z \alpha)^{3} \ln Z \alpha \quad E_{F}, \quad \alpha^{2}(Z \alpha)^{2} \ln ^{2} Z \alpha \quad E_{F}\right.$, $\alpha^{2}(Z \alpha)^{2} \ln Z \alpha E_{F},(Z \alpha)^{3}(m / M) \ln ^{2} Z \alpha E_{F},(Z \alpha)^{3}(m / M) \ln Z \alpha \ln (m / M) E_{F}$,

$\left.\alpha(Z \alpha)^{2}(m / M) \ln ^{2} Z \alpha \quad E_{F}\right)$ were obtained. Some other corrections of higher orders $\left(\alpha^{2}(Z \alpha)(m / M) \ln ^{3}(m / M) E_{F}, \alpha^{2}(Z \alpha)(m / M) \ln ^{2}(m / M) E_{F}\right)$ have already been known for some time [13,[14]. The magnitude of these higher order corrections varies from a few thousandths to a few tenths of a kilohertz and some of them turned out to be rather large. The correction of order $\alpha(Z \alpha)^{2} E_{F}$ was also recalculated recently [15,16.

In this paper we consider radiative-recoil corrections of order $\alpha(Z \alpha)(m / M)^{2} E_{F}$ and $\left(Z^{2} \alpha\right)(Z \alpha)(m / M)^{2} E_{F}$, which were not discussed so far. For muonium both the fine structure constant $\alpha$ and the electron-muon mass ratio $(m / M)$ are of the same order of magnitude, and one could anticipate that the corrections under discussion would produce contributions comparable to the higher order corrections of order $\alpha^{4} E_{F}$ mentioned above. Another reason for interest in the radiative-recoil correction of second order in mass ratio is connected with a long standing discrepancy between the results of [17] and [18,19] for radiative-recoil corrections of the first order in mass ratio, induced by the radiative insertions in the electron line. The discrepancy is about three standard deviations of the accuracy of the numerical calculations in [17, and is equal to $0.22 \mathrm{kHz}$. This discrepancy, which was of a purely academic interest when the respective calculations were performed, now acquires some practical importance. An apparent reason for this contradiction could be connected with the fact that while in [18,19] an explicit expansion in the small mass ratio was performed, with only the contribution of the first order preserved, in the calculations in [17] only the nonrecoil contribution was thrown away, and consequently the result of this work contains terms linear in the mass ratio as well as all contributions of higher orders in the mass ratio. With the results of this work we will be able to test this hypothesis about the origin of the discrepancy between the results in [17] and [18,19].

Radiative-recoil corrections to hyperfine splitting are generated by the diagrams with all possible radiative insertions in the two-photon exchange graphs. It suffices to calculate 
respective matrix elements between the Coulomb-Schrödinger wave functions (see, e.g., discussion in [20]). We will consider below separately the corrections generated by the three types of diagrams: polarization insertions in the exchanged photons, radiative insertions in the electron line, and radiative insertions in the muon line.

\section{POLARIZATION OPERATOR CONTRIBUTIONS}

The general expression for the $\alpha(Z \alpha) E_{F}$ corrections to hyperfine splitting induced by the electron vacuum polarization insertions in the exchanged photons has the form (see, e.g., [20])

$$
\begin{array}{r}
\delta E^{\mathrm{pol}}=\alpha(Z \alpha) E_{F} \frac{1}{\pi^{2} \mu} \int_{0}^{1} d v \int \frac{d^{4} k}{i \pi^{2}} \frac{1}{k^{2}} \frac{v^{2}\left(1-v^{2} / 3\right)}{4-k^{2}\left(1-v^{2}\right)} \\
\left(\frac{1}{k^{2}+\mu^{-1} k_{0}+i 0}+\frac{1}{k^{2}-\mu^{-1} k_{0}+i 0}\right) \frac{3 k_{0}^{2}-2 \mathbf{k}^{2}}{k^{2}-2 k_{0}}
\end{array}
$$

where the exchanged momentum $k$ is measured in units of the electron mass and the dimensionless parameter $\mu$ is given by the expression $\mu=m /(2 M)$. Note that the expression in Eq. (2) differs from the integral for the skeleton graph with two exchanged photons only by the substitution

$$
\frac{1}{k^{2}} \rightarrow \frac{\alpha}{\pi} \int_{0}^{1} d v \frac{v^{2}\left(1-v^{2} / 3\right)}{k^{2}\left(1-v^{2}\right)-4}
$$

Let us now describe briefly the calculation of the contribution of relative order $\mu^{2}$ contained in Eq. (2). First we combine the electron and the photon denominators, and obtain

$$
\begin{gathered}
\delta E^{\mathrm{pol}}=\alpha(Z \alpha) E_{F}\left(-\frac{2}{\pi^{2} \mu}\right) \int_{0}^{1} \frac{d v}{1-v^{2}} v^{2}\left(1-\frac{v^{2}}{3}\right) \int_{0}^{1} d z \\
\left(-\frac{\partial}{\partial a_{p}^{2}}\right) \int \frac{d^{4} k}{i \pi^{2}} \frac{1}{k^{4}-\mu^{-2} k_{0}^{2}} \frac{3 k_{0}^{2}-2 \mathbf{k}^{2}}{\left(-k^{2}+2 b_{p} k_{0}+a_{p}^{2}\right)},
\end{gathered}
$$

where $a_{p}^{2}=4 z /\left(1-v^{2}\right), b_{p}=1-z$.

The general expression in Eq. (四) contains nonrecoil radiative corrections of order $\alpha(Z \alpha) E_{F}$ 21,22, as well as radiative-recoil corrections of all orders in the electron-muon mass ratio generated by the polarization operator insertions and admits numerical calculation. Due to the structure of the integrand explicit extraction of the corrections of definite order in the mass ratio is more involved. Direct application of the standard Feynman parameter methods leads to such integrals for the radiative-recoil corrections which do not admit expansion of the integrand over the small mass ratio before integration, making the analytic calculation virtually impossible. Instead of combining denominators in Eq. (14) with the help of the Feynman parameters we use an approach developed earlier for calculation of the radiative-recoil corrections of order $\alpha(Z \alpha)(m / M) E_{F}$ (see, e.g., review in [20]). The idea 
is to perform integration over the exchanged momentum directly in spherical coordinates. Following this route we come to the expression

$$
\begin{aligned}
& \delta E^{\mathrm{pol}}=\alpha(Z \alpha) E_{F}\left(-\frac{4 \mu}{\pi^{2}}\right) \int_{0}^{1} \frac{d v}{1-v^{2}} v^{2}\left(1-\frac{v^{2}}{3}\right) \int_{0}^{1} d z \\
& \frac{\partial}{\partial a_{p}^{2}}\left\{\int_{0}^{\infty} d k^{2} \frac{k^{2}\left(k^{2}+a_{p}^{2}\right)}{\left(k^{2}+a_{p}^{2}\right)^{2}-4 \mu^{2} b_{p}^{2} k^{4}}\left[2 \Phi_{0}(k)+\Phi_{1}(k)\right]\right\},
\end{aligned}
$$

which readily admits expansion in the small parameter $\mu$. We have introduced here standard auxiliary functions $\Phi_{n}(k)$, which emerge in all calculations in this paper. Definitions and properties of these functions are described in Appendix A.

The crucial property of the momentum integrand in Eq. (5), which facilitates further calculation, is that the denominator admits expansion in the small parameter $\mu$ prior to momentum integration. This is true due to the inequality $4 \mu^{2} b_{p}^{2} k^{4} /\left(k^{2}+a^{2}\right)^{2} \leq 4 \mu^{2}$ which is obviously satisfied because $0 \leq a_{p}<\infty$ and $0 \leq b_{p} \leq 1$. In this way we may easily reproduce nonrecoil radiative corrections induced by the vacuum polarization insertions [21,22], and respective corrections of the first order in the mass ratio which were obtained in [23, 24]. We will omit here these calculations and describe only calculation of the corrections of order $(m / M)^{2}$. Expanding the denominator in Eq. (5) in the small mass ratio we obtain

$$
\begin{gathered}
\delta E^{\mathrm{pol}} \approx \alpha(Z \alpha) E_{F}\left(-\frac{4 \mu}{\pi^{2}}\right) \int_{0}^{1} \frac{d v}{1-v^{2}} v^{2}\left(1-\frac{v^{2}}{3}\right) \int_{0}^{1} d z \\
\frac{\partial}{\partial a_{p}^{2}}\left\{\int_{0}^{\infty} d k^{2} k^{2}\left[\frac{1}{k^{2}+a_{p}^{2}}+\frac{4 \mu^{2} b_{p}^{2} k^{4}}{\left(k^{2}+a_{p}^{2}\right)^{3}}\right]\left[2 \Phi_{0}^{S}(k)+2 \Phi_{0}^{\mu}(k)+\Phi_{1}^{\mu}(k)+2 \Phi_{0}^{C}(k)+\Phi_{1}^{C}(k)\right]\right\} .
\end{gathered}
$$

Functions $\Phi_{i}^{C}(k)$ do not depend on the small parameter $\mu$ (see Appendix A) and, hence, do not generate corrections of order $\mu^{2}$. Calculation of the $\mu$-integrals (integrals with functions $\Phi^{\mu}$ in the integrands) is performed using an auxiliary parameter $\sigma\left(1 \ll \sigma \ll \mu^{-1}\right)$ (see a more detailed discussion in the next Section [III). Detailed analysis demonstrates that only the region of small momenta (in comparison with the inverse electron-muon mass ratio) generates corrections of the second order in $(m / M)^{2}$. All such corrections are connected with the product of the function $\Phi_{0}^{\mu}$ and of the term with factor $b_{p}$ in the numerator in Eq. (6) land we obtain

$$
\delta E_{\mathrm{vp}}=\alpha(Z \alpha) E_{F} \frac{2}{\pi} \int_{0}^{1} \frac{d v}{1-v^{2}} v^{2}\left(1-\frac{v^{2}}{3}\right) \int_{0}^{1} \frac{d z}{a_{p}} 15 \mu^{2} b_{p}^{2}=\frac{3}{4} \alpha(Z \alpha)\left(\frac{m}{M}\right)^{2} E_{F} .
$$

Besides the electron vacuum polarization one also has to consider the muon vacuum polarization insertion. The respective contribution differs from the one for the electron only by the substitution $k \rightarrow(M / m) k$ and has the form

\footnotetext{
${ }^{1}$ All statements above are proved in Appendix B.
} 


$$
\begin{aligned}
\delta E_{\mu \mathrm{vp}}= & \alpha(Z \alpha) E_{F} \frac{16 \mu}{\pi^{3}} \int_{0}^{1} d v v^{2}\left(1-\frac{v^{2}}{3}\right) \int_{0}^{\pi} d \theta \sin ^{2} \theta\left(2+\cos ^{2} \theta\right) \\
& \int_{0}^{\infty} d k^{2} \frac{1}{k^{2}+4 \cos ^{2} \theta} \frac{1}{4+k^{2}\left(1-v^{2}\right)} \frac{k^{2}}{k^{2}+16 \mu^{2} \cos ^{2} \theta} .
\end{aligned}
$$

Representing the last factor in the integrand in the form

$$
\frac{k^{2}}{k^{2}+16 \mu^{2} \cos ^{2} \theta} \equiv 1-\frac{16 \mu^{2} \cos ^{2} \theta}{k^{2}+16 \mu^{2} \cos ^{2} \theta},
$$

we easily obtain

$$
\delta E_{\mu \mathrm{vp}}=\left(\frac{\pi^{2}}{3}-\frac{10}{9}\right) \frac{\alpha(Z \alpha)}{\pi^{2}}\left(\frac{m}{M}\right) E_{F}+O\left(\mu^{3}\right)
$$

and, hence, the muon vacuum polarization does not generate contributions of order $(m / M)^{2}$. Hadron vacuum polarization insertions lead to the corrections of the same order as the muon vacuum polarization and will be ignored in our discussion.

\section{ELECTRON-LINE CONTRIBUTION}

Radiative corrections to the hyperfine splitting induced by the radiative insertions in the electron line are given by the expression [19]

$$
\begin{gathered}
\delta E^{\mathrm{e}-\text { line }}=\frac{Z \alpha}{\pi} E_{F}\left(-\frac{3}{16 \mu}\right) \int \frac{d^{4} k}{i \pi^{2}} \frac{1}{\left(k^{2}+i 0\right)^{2}}\left(\frac{1}{k^{2}+\mu^{-1} k_{0}+i 0}\right. \\
\left.+\frac{1}{k^{2}-\mu^{-1} k_{0}+i 0}\right)\left\langle\gamma^{\mu} \hat{k} \gamma^{\nu}\right\rangle_{(\mu)} L_{\mu \nu},
\end{gathered}
$$

where the electron factor $L_{\mu \nu}$ is a sum of four terms $L_{\mu \nu}=\sum_{1}^{4} L_{\mu \nu}^{i}$ [19], which after some transformations may be written in the form

$$
\begin{gathered}
L_{\mu \nu}^{(1)}=\frac{\alpha}{4 \pi}\left\langle\gamma_{\mu} \hat{k} \gamma_{\nu}\right\rangle_{(e)} \int_{0}^{1} d x \int_{0}^{x} \frac{d y}{\left(-k^{2}+2 b k_{0}+a^{2}\right)^{3}}\left\{c_{1} \mathbf{k}^{2}+c_{2} k^{4}\right\}, \\
L_{\mu \nu}^{(2)}=\frac{\alpha}{4 \pi}\left\langle\gamma_{\mu} \hat{k} \gamma_{\nu}\right\rangle_{(e)} \int_{0}^{1} d x \int_{0}^{x} \frac{d y}{\left(-k^{2}+2 b k_{0}+a^{2}\right)^{2}}\left\{c_{3} k^{2}+2 c_{4} k_{0}\right\}, \\
L_{\mu \nu}^{(3)}=\frac{\alpha}{4 \pi}\left\langle\gamma_{\mu} \gamma_{\nu}\right\rangle_{(e)} \int_{0}^{1} d x \int_{0}^{x} \frac{d y}{\left(-k^{2}+2 b k_{0}+a^{2}\right)^{2}}\left\{c_{5} k^{2}+2 c_{6} k^{2} k_{0}\right\}, \\
L_{\mu \nu}^{(4)}=\frac{\alpha}{4 \pi}\left\langle\gamma_{\mu} \gamma_{\nu}\right\rangle_{(e)} \int_{0}^{1} d x \int_{0}^{x} \frac{d y}{-k^{2}+2 b k_{0}+a^{2}}\left\{c_{7} k^{2}\right\} .
\end{gathered}
$$


Auxiliary functions of the Feynman parameters $a(x, y)$ and $b(x, y)$ are defined by the relationships

$$
a^{2}=\frac{x^{2}}{y(1-y)}, \quad b=\frac{1-x}{1-y}
$$

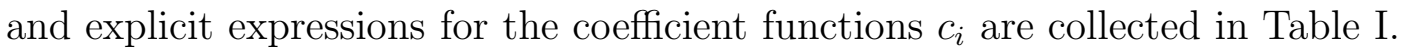
Performing contractions of the products of the electron and muon spinor structures

$$
\begin{gathered}
\left\langle\gamma_{\mu} \hat{k} \gamma_{\nu}\right\rangle_{(e)}\left\langle\gamma^{\mu} \hat{k} \gamma^{\nu}\right\rangle_{(\mu)}=\frac{8}{3}\left(-3 k_{0}^{2}+2 \mathbf{k}^{2}\right), \\
\left\langle\gamma_{\mu} \gamma_{\nu}\right\rangle_{(e)}\left\langle\gamma^{\mu} \hat{k} \gamma^{\nu}\right\rangle_{(\mu)}=8 k_{0}
\end{gathered}
$$

we arrive at the expression for the electron line contribution to the hyperfine splitting

$$
\begin{gathered}
\delta E^{\mathrm{e}-\text { line }}=\alpha(Z \alpha) E_{F} \frac{1}{8 \pi^{2} \mu} \int_{0}^{1} d x \int_{0}^{x} d y \int \frac{d^{4} k}{i \pi^{2}} \frac{1}{\left(k^{2}+i 0\right)^{2}} \\
\left(\frac{1}{k^{2}+\mu^{-1} k_{0}+i 0}+\frac{1}{k^{2}-\mu^{-1} k_{0}+i 0}\right) \\
\left\{\left(3 k_{0}^{2}-2 \mathbf{k}^{2}\right)\left[\frac{c_{1} \mathbf{k}^{2}+c_{2}\left(k^{2}\right)^{2}}{\left(-k^{2}+2 b k_{0}+a^{2}\right)^{3}}+\frac{c_{3} k^{2}+c_{4} 2 k_{0}}{\left(-k^{2}+2 b k_{0}+a^{2}\right)^{2}}\right]\right. \\
\left.-3 k_{0}\left[\frac{c_{5} k^{2}+c_{6} k^{2} 2 k_{0}}{\left(-k^{2}+2 b k_{0}+a^{2}\right)^{2}}+\frac{c_{7} k^{2}}{-k^{2}+2 b k_{0}+a^{2}}\right]\right\} \equiv \sum_{1}^{7} \delta E_{i} .
\end{gathered}
$$

Now we will discuss briefly calculation of the contributions to the hyperfine splitting of order $\alpha(Z \alpha)(m / M)^{2} E_{F}$ generated by the electron factor. Let us start our consideration with the simplest contribution corresponding to the coefficient function $c_{7}$ in Table $\mathbb{E}$. After Wick rotation and calculation of the angle integral we arrive at the expression

$$
\delta E=\alpha(Z \alpha) E_{F}\left(-\frac{3 \mu}{\pi^{2}}\right) \int_{0}^{1} d x \int_{0}^{x} d y b c_{7} \int_{0}^{\infty} d k^{2} \frac{k^{2}}{\left(k^{2}+a^{2}\right)^{2}-4 \mu^{2} b^{2} k^{4}} \Phi_{1}(k),
$$

where the auxiliary function $\Phi_{1}(k)$ is defined in the Appendix A.

As in the case of the vacuum polarization discussed above one may expand the denominator of the integrand and obtain

$$
\begin{gathered}
\delta E \approx \alpha(Z \alpha) E_{F}\left(-\frac{3 \mu}{\pi^{2}}\right) \int_{0}^{1} d x \int_{0}^{x} d y b c_{7} \\
\int_{0}^{\infty} d k^{2} \frac{k^{2}}{\left(k^{2}+a^{2}\right)^{2}}\left\{1+\frac{4 \mu^{2} b^{2} k^{4}}{\left(k^{2}+a^{2}\right)^{2}}\right\}\left[\Phi_{1}^{\mu}(k)+\Phi_{1}^{C}(k)\right],
\end{gathered}
$$


where the second term in the braces is at least of the second order in $\mu^{2}$ due to an obvious inequality $4 \mu^{2} b^{2} k^{4} /\left(k^{2}+a^{2}\right)^{2} \leq 4 \mu^{2}$.

It is easy to see that the $c$-integral (integral which contains function $\Phi_{1}^{C}$ in the integrand) does not generate corrections of the second order in $\mu^{2}$ since the function $\Phi_{1}^{C}$ is $\mu$-independent. Only the $\mu$-integral

$$
\delta E_{7}^{\mu}=\alpha(Z \alpha) E_{F}\left(-\frac{3 \mu}{\pi^{2}}\right) \int_{0}^{1} d x \int_{0}^{x} d y b c_{7} \int_{0}^{\infty} d k^{2} \frac{k^{2}}{\left(k^{2}+a^{2}\right)^{2}} \Phi_{1}^{\mu}(k),
$$

generates corrections of the second order in the mass ratio.

As we already mentioned in Section II the integrals of this kind are usually calculated with the help of an auxiliary parameter $\sigma$, which satisfies the inequality $1 \ll \sigma \ll \mu^{-1}$. The parameter $\sigma$ is used to separate the momentum integration into two regions, a region of small momenta $0 \leq k \leq \sigma$, and a region of large momenta $\sigma \leq k<\infty$. In the region of small momenta one uses for simplification of the integrand the condition $\mu k \ll 1$, and in the region of large momenta the condition $k \gg 1$. Note that for $k \simeq \sigma$ both conditions on the integration momenta are valid simultaneously, so in the sum of the low-momenta and high-momenta integrals all $\sigma$-dependent terms cancel and one obtains a $\sigma$-independent result for the total momentum integral (see more detailed discussion of this method, e.g., in [20]). Sometimes one needs to use more stringent restrictions on the auxiliary parameter $\sigma$ in order to obtain a result with higher accuracy in $\mu$. This case, as well as the sample calculation of the $\mu$-integral for the $\delta E_{7}$ contribution are discussed in more detail in Appendix $\mathbb{B}$. The analysis performed in this Appendix leads to the conclusion that all corrections of order $(m / M)^{2}$ are generated by the low-momentum part of the $\mu$-integral in Eq. (18), and in order to obtain these corrections one simply has to extract that contribution of order $\mu^{2}$ to this integral which remains finite when the auxiliary parameter $\sigma$ goes to infinity. Then it is easy to obtain the respective contribution

$$
\delta E_{7}=\alpha(Z \alpha) E_{F}\left(-\frac{9 \mu^{2}}{2 \pi}\right) \int_{0}^{1} d x \int_{0}^{x} d y b c_{7} a=-\frac{9}{32} \alpha(Z \alpha)\left(\frac{m}{M}\right)^{2} E_{F} .
$$

Calculation of the contribution to the hyperfine splitting induced by the term with the coefficient function $c_{5}$ in Eq. (15) proceeds essentially along the same lines as in the case of the $\delta E_{7}$ contribution. Again the $c$-integral does not generate corrections of order $\mu^{2}$ to the hyperfine splitting. An integral representation for the $\mu$-integral contribution may be obtained from the representation in Eq. (18) by the substitution $c_{7} \rightarrow-c_{5}\left(\partial /\left(\partial a^{2}\right)\right)$

$$
\delta E_{5}^{\mu}=\alpha(Z \alpha) E_{F}\left(-\frac{6 \mu}{\pi^{2}}\right) \int_{0}^{1} d x \int_{0}^{x} d y b c_{5} \int_{0}^{\infty} d k^{2} \frac{k^{2}}{\left(k^{2}+a^{2}\right)^{3}} \Phi_{1}^{\mu}(k) .
$$

Correction of order $\mu^{2}$ to the hyperfine splitting is generated by the term linear in $\mu$ in the low momentum expansion of the function $\Phi_{1}^{\mu}(k)$. Due to a higher power of the denominator in the integrand in Eq. (20) than in Eq. (18) the respective momentum integral is ultraviolet convergent and we immediately obtain

$$
\delta E_{5}=\alpha(Z \alpha) E_{F} \frac{9 \mu^{2}}{4 \pi} \int_{0}^{1} d x \int_{0}^{x} d y \frac{b c_{5}}{a}=-\frac{3}{32} \alpha(Z \alpha)\left(\frac{m}{M}\right)^{2} E_{F} .
$$


In the case of the weight function $c_{6}$ in Eq. (15) the $\mu$-integral has the form

$$
\delta E_{6}^{\mu}=\alpha(Z \alpha) E_{F} \frac{3 \mu}{\pi^{2}} \int_{0}^{1} d x \int_{0}^{x} d y c_{6} \int_{0}^{\infty} d k^{2} \frac{k^{2}}{\left(k^{2}+a^{2}\right)^{2}} \Phi_{1}^{\mu}(k) .
$$

Again extracting the term linear in $\mu$ from the integrand we obtain

$$
\delta E_{6}=\alpha(Z \alpha) E_{F} \frac{9 \mu^{2}}{2 \pi} \int_{0}^{1} d x \int_{0}^{x} d y c_{6} a=-\frac{3}{32} \alpha(Z \alpha)\left(\frac{m}{M}\right)^{2} E_{F} .
$$

Unlike all cases discussed above the $\mu$-integral for the weight function $c_{4}$ depends not only on the function $\Phi_{1}^{\mu}(k)$ but also on the function $\Phi_{2}^{\mu}(k)$

$$
\delta E_{4}^{\mu}=\alpha(Z \alpha) E_{F} \frac{4 \mu}{\pi^{2}} \int_{0}^{1} d x \int_{0}^{x} d y b c_{4} \int_{0}^{\infty} d k^{2} \frac{k^{2}}{\left(k^{2}+a^{2}\right)^{3}}\left[2 \Phi_{1}^{\mu}(k)+\Phi_{2}^{\mu}(k)\right] .
$$

However, the conclusion of Appendix $\mathbb{B}$ that only the low-momentum part of the $\mu$ integrals leads to corrections of order $\mu^{2}$ to hyperfine splitting remains valid, even in this case. We may repeat the considerations of Appendix B for this case, and check that the additional high-momentum contributions to the integral generated by the auxiliary high momentum integrals $V_{202}$ and $V_{212}$ (see definitions of these integrals and discussion of their properties in Appendix (9) differ from the respective contributions $V_{201}$ and $V_{211}$ connected with the function $\Phi_{1}^{\mu}(k)$ only by the factor $1 / 4$. Hence they do not lead to corrections of order $\mu^{2}$ to the hyperfine splitting. Moreover, terms linear in $\mu$ are missing in the small momentum expansion of the function $\Phi_{2}^{\mu}(k)$. Hence, one may completely ignore presence of the function $\Phi_{2}^{\mu}(k)$ in the integrand in Eq. (24) during calculation of the contribution to the hyperfine splitting of relative order $\mu^{2}$. Then calculation of the contribution $\delta E_{4}$ may be done exactly in the same way as calculation of the contribution $\delta E_{5}$ above and one obtains

$$
\delta E_{4}=\alpha(Z \alpha) E_{F}\left(-\frac{3 \mu^{2}}{\pi}\right) \int_{0}^{1} d x \int_{0}^{x} d y \frac{b c_{4}}{a}=\frac{3}{16} \alpha(Z \alpha)\left(\frac{m}{M}\right)^{2} E_{F} .
$$

We will combine consideration of the contributions to hyperfine corresponding to the weight factors $c_{1}, c_{2}$, and $c_{3}$. In this case, after the angular integration, we arrive at the expression

$$
\begin{gathered}
\delta E=\alpha(Z \alpha) E_{F} \frac{\mu}{4 \pi^{2}} \int_{0}^{1} d x \int_{0}^{x} d y \int_{0}^{\infty} d k^{2} k^{2}\left\{\left[\left(c_{1}+c_{2} k^{2}\right)\left(\frac{\partial}{\partial a^{2}}\right)^{2}+2 c_{3} \frac{\partial}{\partial a^{2}}\right]\right. \\
\left.\frac{k^{2}+a^{2}}{\left(k^{2}+a^{2}\right)^{2}-4 \mu^{2} b^{2} k^{4}}\left[2 \Phi_{0}(k)+\Phi_{1}(k)\right]-c_{1}\left(\frac{\partial}{\partial a^{2}}\right)^{2} \frac{k^{2}+a^{2}}{\left(k^{2}+a^{2}\right)^{2}-4 \mu^{2} b^{2} k^{4}}\left[2 \Phi_{1}(k)+\Phi_{2}(k)\right]\right\} .
\end{gathered}
$$

Differentiation and expansion in a series in $\mu^{2}$ leads to the integral

$$
\begin{aligned}
& \delta E \approx \alpha(Z \alpha) E_{F} \frac{\mu}{2 \pi^{2}} \int_{0}^{1} d x \int_{0}^{x} d y \int_{0}^{\infty} d k^{2} k^{2}\left\{\left[\frac{c_{1}+c_{2} k^{2}}{\left(k^{2}+a^{2}\right)^{3}}-\frac{c_{3}}{\left(k^{2}+a^{2}\right)^{2}}\right]\right. \\
& {\left[2 \Phi_{0}(k)+\Phi_{1}(k)\right]+12 \mu^{2} b^{2} k^{4}\left[\frac{2 c_{1}+2 c_{2} k^{2}}{\left(k^{2}+a^{2}\right)^{5}}-\frac{c_{3}}{\left(k^{2}+a^{2}\right)^{4}}\right]\left[2 \Phi_{0}(k)+\Phi_{1}(k)\right]}
\end{aligned}
$$




$$
\left.-c_{1}\left[\frac{1}{\left(k^{2}+a^{2}\right)^{3}}+\frac{24 \mu^{2} b^{2} k^{4}}{\left(k^{2}+a^{2}\right)^{5}}\right]\left[2 \Phi_{1}(k)+\Phi_{2}(k)\right]\right\} .
$$

As usual we write the functions $\Phi_{i}(k)$ as sums of the functions $\Phi_{i}^{\mu}(k)$ and $\Phi_{i}^{C}(k)$ (see Appendix B). Let us mention here that the classical nonrecoil electron-factor contribution to the hyperfine splitting [21,22] is produced by the term with $\Phi_{0}^{S}=2 /(\mu k)$ in the integral above

$$
\begin{gathered}
\delta E_{K P}=\alpha(Z \alpha) E_{F} \frac{2}{\pi^{2}} \int_{0}^{1} d x \int_{0}^{x} d y \int_{0}^{\infty} d k k^{2}\left[\frac{c_{1}+c_{2} k^{2}}{\left(k^{2}+a^{2}\right)^{3}}-\frac{c_{3}}{\left(k^{2}+a^{2}\right)^{2}}\right] \\
=\alpha(Z \alpha) E_{F}\left(\ln 2-\frac{13}{4}\right) .
\end{gathered}
$$

Contributions of relative order $\mu^{2}$ are connected only with the functions $\Phi_{i}^{\mu}$, and are hidden in the integral

$$
\begin{gathered}
\delta E_{123}^{\mu} \approx \alpha(Z \alpha) E_{F} \frac{\mu}{2 \pi^{2}} \int_{0}^{1} d x \int_{0}^{x} d y \int_{0}^{\infty} d k^{2} k^{2}\left\{[ 2 \Phi _ { 0 } ^ { \mu } ( k ) + \Phi _ { 1 } ^ { \mu } ( k ) ] \left[\frac{c_{1}+c_{2} k^{2}}{\left(k^{2}+a^{2}\right)^{3}}-\right.\right. \\
\left.\left.\frac{c_{3}}{\left(k^{2}+a^{2}\right)^{2}}\right]+24 \mu^{2} b^{2} k^{4} \Phi_{0}^{S}(k)\left[\frac{2 c_{1}+2 c_{2} k^{2}}{\left(k^{2}+a^{2}\right)^{5}}-\frac{c_{3}}{\left(k^{2}+a^{2}\right)^{4}}\right]-\left[2 \Phi_{1}^{\mu}(k)+\Phi_{2}^{\mu}(k)\right] \frac{c_{1}}{\left(k^{2}+a^{2}\right)^{3}}\right\} .
\end{gathered}
$$

As usual, all contributions of relative order $\mu^{2}$ are generated by the low-momentum integration region $0 \leq k \leq \sigma$, where one can use the small momentum approximation for the functions $\Phi_{i}^{\mu}$

$$
\begin{aligned}
2 \Phi_{0}^{\mu}(k)+\Phi_{1}^{\mu}(k) & \approx-\frac{3}{2}+(\mu k)^{2}, \\
2 \Phi_{1}^{\mu}(k)+\Phi_{2}^{\mu}(k) & \approx \frac{9}{8}-2 \mu k+\frac{3}{2}(\mu k)^{2} .
\end{aligned}
$$

Due to absence of terms linear in $\mu$ in the low-momentum expansion of the function $2 \Phi_{0}^{\mu}(k)+\Phi_{1}^{\mu}(k)$, the respective term in the integrand in Eq. (29) does not generate corrections of relative order $\mu^{2}$. Hence all such corrections in Eq. (29) are connected with the functions $\Phi_{0}^{S}(k)=1 / \mu k$ and $2 \Phi_{1}^{\mu}(k)+\Phi_{2}^{\mu}(k) \approx-2 \mu k$, and are given by the expression

$$
\begin{gathered}
\delta E_{123}=\alpha(Z \alpha) E_{F} \frac{2 \mu^{2}}{\pi^{2}} \int_{0}^{1} d x \int_{0}^{x} d y \int_{0}^{\infty} d k\left\{\frac{c_{1} k^{4}}{\left(k^{2}+a^{2}\right)^{3}}+\right. \\
\left.12 b^{2} k^{6}\left[\frac{2 c_{1}+2 c_{2} k^{2}}{\left(k^{2}+a^{2}\right)^{5}}-\frac{c_{3}}{\left(k^{2}+a^{2}\right)^{4}}\right]\right\}=\left(-6 \ln 2-\frac{39}{32}\right) \alpha(Z \alpha)\left(\frac{m}{M}\right)^{2} E_{F} .
\end{gathered}
$$

Now we are in a position to write down the total contribution to the hyperfine splitting of relative order $\mu^{2}$ generated by the electron factor, which is given by the sum of the contributions in Eq. (19), Eq. (21), Eq. (23), Eq. (25), and Eq. (31) (see also Table II)

$$
\delta E_{\mathrm{el}}=\left(-6 \ln 2-\frac{3}{2}\right) \alpha(Z \alpha)\left(\frac{m}{M}\right)^{2} E_{F} .
$$




\section{MUON-LINE CONTRIBUTION}

After an obvious substitution $\alpha \rightarrow Z^{2} \alpha$ the electron-line factor in Eq. (12) properly describes radiative corrections to the muon line as well. Then the total expression for the muon-line contribution to hyperfine splitting of order $\left(Z^{2} \alpha\right)(Z \alpha) E_{F}$ may be easily obtained from Eq. (11) and has the form

$$
\begin{gathered}
\delta E^{\mu-\text { line }}=\left(Z^{2} \alpha\right)(Z \alpha) E_{F} \frac{\mu}{2 \pi^{2}} \int_{0}^{1} d x \int_{0}^{x} d y \int \frac{d^{4} k}{i \pi^{2}} \frac{1}{\left(k^{2}+i 0\right)^{2}}\left(\frac{1}{k^{2}+4 \mu k_{0}+i 0}\right. \\
\left.+\frac{1}{k^{2}-4 \mu k_{0}+i 0}\right)\left\{\left(3 k_{0}^{2}-2 \mathbf{k}^{2}\right)\left[\frac{c_{1} \mathbf{k}^{2}+c_{2}\left(k^{2}\right)^{2}}{\left(-k^{2}+2 b k_{0}+a^{2}\right)^{3}}+\frac{c_{3} k^{2}+c_{4} 2 k_{0}}{\left(-k^{2}+2 b k_{0}+a^{2}\right)^{2}}\right]\right. \\
\left.-3 k_{0}\left[\frac{c_{5} k^{2}+c_{6} k^{2} 2 k_{0}}{\left(-k^{2}+2 b k_{0}+a^{2}\right)^{2}}+\frac{c_{7} k^{2}}{-k^{2}+2 b k_{0}+a^{2}}\right]\right\} .
\end{gathered}
$$

Apparent distinctions between Eq. (11) and Eq. (33) remind us that while in Eq. (11) the dimensionless integration momentum is measured in the units of electron mass, in Eq. (33) it is measured in the muon mass units.

After standard transformations the integral in Eq. (33) acquires the form

$$
\delta E^{\mu-\text { line }}=\left(Z^{2} \alpha\right)(Z \alpha) E_{F} \frac{\mu}{\pi^{3}} \int_{0}^{\pi} d \theta \sin ^{2} \theta \int_{0}^{\infty} d k^{2}\left(1-\frac{16 \mu^{2} \cos ^{2} \theta}{k^{2}+16 \mu^{2} \cos ^{2} \theta}\right) I(k),
$$

where the first factor in the integrand is simplified with the help of the identity in Eq. (9), and the second factor is given by the integral

$$
\begin{aligned}
I(k)=\int_{0}^{1} d x & \int_{0}^{x} d y\left\{\left(2+\cos ^{2} \theta\right)\left[\left(c_{1} \sin ^{2} \theta+c_{2} k^{2}\right)\left(\frac{\partial}{\partial a^{2}}\right)^{2}+2 c_{3} \frac{\partial}{\partial a^{2}}\right]\left(k^{2}+a^{2}\right)\right. \\
& -8 b c_{4}\left(2+\cos ^{2} \theta\right) \cos ^{2} \theta \frac{\partial}{\partial a^{2}}+12 b \cos ^{2} \theta\left(c_{5} \frac{\partial}{\partial a^{2}}-c_{7}\right) \\
& \left.-12 c_{6} \cos ^{2} \theta \frac{\partial}{\partial a^{2}}\left(k^{2}+a^{2}\right)\right\} \frac{1}{\left(k^{2}+a^{2}\right)^{2}+4 b^{2} k^{2} \cos ^{2} \theta} .
\end{aligned}
$$

The first factor in the parenthesis in the integrand in Eq. (34) generates only corrections of relative order $\mu$, since the integral $I(k)$ is $\mu$-independent. The respective correction to hyperfine splitting was calculated long time ago [17,25,26], and we will not consider it here. Correction of order $\mu^{2}$ in Eq. (34) could be connected only with second term in the parenthesis which apparently generates a contribution of order $\mu^{3}$. However, the integral $I(k)$ behaves like $1 / k$ at small momenta, and this singular behavior reduces the apparent power of $\mu$, generating a correction of relative order $\mu^{2}$. In order to obtain this correction we have to calculate the leading term in the low-momentum expansion of the integral $I(k)$. 
Let us outline briefly the respective calculation. First, we note that the most singular $k$ behavior of the integral in Eq. (35) is connected with the region of small values of the Feynman parameter $x$. Hence, in the limit of small $k$ and $x$ we preserve in the integrand only nonvanishing terms. The calculation is facilitated by the change of variable $y=x z$. Then use the following approximations

$$
\begin{gathered}
a^{2} \approx \frac{x}{z}, \quad b \approx 1, \quad k^{2}+a^{2} \approx \frac{x}{z} \\
c_{1} \approx 16\left(\frac{1}{z}-3-2 \ln x\right), \quad c_{3} \approx \frac{1}{x}\left(\frac{1}{z}-26+6 z-16 \ln x\right), \\
c_{4} \approx \frac{2}{z}-5, \quad c_{5} \approx \frac{6}{z}-8, \quad c_{6} \approx-\frac{1-z}{x}, \quad c_{7} \approx \frac{2}{x}
\end{gathered}
$$

The term with the coefficient function $c_{2}$ in Eq. (35) may be omitted since it is explicitly multiplied by the small factor $k^{2}$. Then the leading infrared contribution to the function $I(k)$ may be written as

$$
\begin{aligned}
& I(k) \approx \int_{0}^{1} d z z^{2} \int_{0}^{1} d x\left\{( 2 + \operatorname { c o s } ^ { 2 } \theta ) \left[16 \sin ^{2} \theta z\left(\frac{1}{z}-3-2 \ln x\right) x\left(\frac{\partial}{\partial x}\right)^{2} x\right.\right. \\
& \left.+2\left(\frac{1}{z}-26+6 z-16 \ln x\right) \frac{\partial}{\partial x} x\right]+8\left(2+\cos ^{2} \theta\right) \cos ^{2} \theta(-2+5 z) x \frac{\partial}{\partial x} \\
& \left.+12 \cos ^{2} \theta\left[(6-8 z) x \frac{\partial}{\partial x}-2\right]+12 \cos ^{2} \theta(1-z) \frac{\partial}{\partial x} x\right\} \frac{1}{x^{2}+4 k^{2} z^{2} \cos ^{2} \theta} .
\end{aligned}
$$

Integrating by parts we reduce all infrared-hard terms to the standard integral

$$
\int_{0}^{1} \frac{d x}{x^{2}+4 k^{2} z^{2} \cos ^{2} \theta} \approx \frac{\pi}{4 k z \cos \theta}
$$

and arrive at the relationship

$$
\begin{gathered}
I(k) \approx \int_{0}^{1} d z z^{2}\left[32\left(2+\cos ^{2} \theta\right)\left(1-z \sin ^{2} \theta\right)+8\left(2+\cos ^{2} \theta\right) \cos ^{2} \theta(2-5 z)\right. \\
\left.-12 \cos ^{2} \theta(6-8 z+2)\right] \frac{\pi}{4 k z \cos \theta}=\frac{4 \pi}{3} \frac{2+\cos ^{4} \theta}{k \cos \theta}
\end{gathered}
$$

Now we easily obtain radiative-recoil correction of the relative order $\mu^{2}$ from the second term in the integrand in Eq. (34)

$$
\delta E_{\mathrm{ml}}=-\frac{17}{12}\left(Z^{2} \alpha\right)(Z \alpha)\left(\frac{m}{M}\right)^{2} E_{F}
$$




\section{DISCUSSION OF RESULTS}

Let us consider first the electron-line contribution in Eq. (32). This result demonstrates that the discrepancy between the results in [17] and in [18, 19] for the radiative-recoil corrections induced by the electron line cannot be explained by the contributions of the terms of higher order in the mass ratio to the result in [17]. Numerically we obtain from Eq. (32)

$$
\delta E_{\mathrm{el}} \approx-0.03 \mathrm{kHz}
$$

which is too small to explain the difference $0.22 \mathrm{kHz}$ between the results in [17 and [18,19. We would like to remind the reader that the coinciding results in [18 and 19] were obtained completely independently in different gauges, so there is little, if any, doubt that this result is correct.

The total one-loop radiative-recoil correction of relative order $(m / M)^{2}$ is given by the sum of the results in Eq. (7), Eq. (32), and Eq. (40)

$$
\delta E_{\text {rad-rec }}=\left[\left(-6 \ln 2-\frac{3}{4}\right) \alpha(Z \alpha)-\frac{17}{12}\left(Z^{2} \alpha\right)(Z \alpha)\right]\left(\frac{m}{M}\right)^{2} E_{F},
$$

or, recalling that $Z=1$ for muonium

$$
\delta E_{\text {rad-rec }}=\left(-6 \ln 2-\frac{13}{6}\right) \alpha^{2}\left(\frac{m}{M}\right)^{2} E_{F} \approx-6.32555 \ldots \alpha^{2}\left(\frac{m}{M}\right)^{2} E_{F} .
$$

Let us mention a minor subtlety connected with the definition of the Fermi energy in Eq. (42). As is well known, there are two common definitions for the Fermi energy: one includes the muon anomalous magnetic moment, and the other does not. Both definitions are useful in presenting results for the high order corrections to the hyperfine splitting. Corrections, generated at the scale of order $1 / m$ or larger (small intermediate momenta) are multiplied by the Fermi energy which includes the muon anomalous momentum. But for the corrections generated at the scale comparable with the muon Compton length $1 / M$ the muon anomalous momentum cannot be factorized, and such corrections are multiplied by the Fermi energy without the muon anomalous moment. As we have seen above, radiative-recoil corrections of the second order in mass ratio originate from the distances larger than the electron Compton length, and, hence, the Fermi energy in Eq. (42) should include the muon anomalous moment. In this way we properly take into account some corrections of higher order than $\alpha(Z \alpha)(m / M) E_{F}$ but due to the smallness of the correction under consideration this is irrelevant from the purely phenomenological point of view.

Numerically the contribution to hyperfine splitting obtained in this paper is equal to

$$
\delta E_{\text {rad-rec }}=-0.0351 \ldots \mathrm{kHz}
$$

and should be taken into account, along with some other small corrections to hyperfine splitting calculated recently, when comparing the pending experimental results with the theory.

\section{ACKNOWLEDGMENTS}

M. E. and V.S. are deeply grateful for the kind hospitality of the Physics Department at Penn State University, where this work was performed. The authors appreciate the support of this work by the National Science Foundation under grant number PHY-9421408. 


\section{APPENDIX A: STANDARD AUXILIARY FUNCTIONS}

All contributions to hyperfine splitting in the main body of this paper are written in terms of the auxiliary functions $\Phi_{n}(k) \quad(n=0,1,2,3)$ defined by the relationship

$$
\Phi_{n}(k) \equiv \frac{1}{\pi \mu^{2}} \int_{0}^{\pi} d \theta \sin ^{2} \theta \cos ^{2 n} \theta \frac{\left(k^{2}+a^{2}\right)^{2}-4 \mu^{2} b^{2} k^{4}}{\left(k^{2}+\mu^{-2} \cos ^{2} \theta\right)\left[\left(k^{2}+a^{2}\right)^{2}+4 b^{2} k^{2} \cos ^{2} \theta\right]}
$$

The integral over angles may be explicitly calculated, and the result of the integration is conveniently written as a sum

$$
\Phi_{n}(k) \equiv \Phi_{n}^{S}(k)+\Phi_{n}^{\mu}(k)+\Phi_{n}^{C}(k),
$$

where

$$
\begin{gathered}
\Phi_{n}^{S}(k)=\frac{\delta_{n 0}}{\mu k}, \\
\Phi_{0}^{\mu}(k)=W\left(\xi_{\mu}\right)-\frac{1}{\sqrt{\xi_{\mu}}}, \\
\Phi_{1}^{\mu}(k)=-\xi_{\mu} W\left(\xi_{\mu}\right)+\frac{1}{2}, \\
\Phi_{3}^{\mu}(k)=-\xi_{\mu}^{\mu}\left(\xi_{\mu}\left(\xi_{\mu} W\left(\xi_{\mu}\right)-\frac{1}{2}\right)+\frac{1}{8}\right]+\frac{1}{16}, \\
\Phi_{3}^{C}(k)=\xi_{C}^{C}\left[\xi_{C}\left(\xi_{C} W\left(\xi_{\mu}\right)-\frac{1}{2}\right)+\frac{1}{8},\right. \\
\Phi_{1}^{C}(k)=\xi_{C} W\left(\xi_{C}\right)-\frac{1}{2}, \\
-\xi_{C}\left(\xi_{C} W\left(\xi_{C}\right)-\frac{1}{2}\right)-\frac{1}{8}, \\
=\frac{1}{16} .
\end{gathered}
$$

The standard function $W(\xi)$ has the form 


$$
W(\xi)=\sqrt{1+\frac{1}{\xi}}-1 .
$$

and

$$
\xi_{\mu}=\mu^{2} k^{2}, \quad \xi_{C}=\frac{\left(k^{2}+a^{2}\right)^{2}}{4 b^{2} k^{2}}
$$

One may easily obtain asymptotic expressions for the function $W(\xi)$

$$
\begin{aligned}
& \lim _{\xi \rightarrow 0} W(\xi) \rightarrow \frac{1}{\sqrt{\xi}}, \\
& \lim _{\xi \rightarrow \infty} W(\xi) \rightarrow \frac{1}{2 \xi} .
\end{aligned}
$$

High- and low-momentum asymptotic expressions for the functions $\Phi_{i}(k)$ also may be easily calculated. Let us cite low-momentum expansions, which where used in the main text for calculation of the contributions to the hyperfine splitting of relative order $\mu^{2}$

$$
\begin{gathered}
\Phi_{0}^{\mu}(k) \approx-1+\frac{\mu k}{2}, \\
\Phi_{1}^{\mu}(k) \approx \frac{1}{2}-\mu k+(\mu k)^{2}, \\
\Phi_{2}^{\mu}(k) \approx \frac{1}{8}-\frac{(\mu k)^{2}}{2} .
\end{gathered}
$$

\section{APPENDIX B: CALCULATION OF $\mu$-INTEGRALS}

In this Appendix we will consider the main tricks used in calculation of the $\mu$-integrals. Consider as an example calculation of the integral relevant for the $\delta E_{7}$ correction

$$
F(\mu)=\int_{0}^{1} d x \int_{0}^{x} d y b c_{7} \int_{0}^{\infty} d k^{2} \frac{k^{2}}{\left(k^{2}+a^{2}\right)^{2}} \Phi_{1}^{\mu}(k) .
$$

We need to calculate contributions order $\mu$ to this integral. As was discussed in the main text we introduce an auxiliary parameter $\sigma\left(1 \ll \sigma \ll \mu^{-1}\right)$ and consider separately contributions of the large and small integration momenta

$$
F(\mu)=F^{<}(\mu)+F^{>}(\mu) .
$$

In the large momenta region we would like to use the relationship $k \geq \sigma \gg 1$ in order to simplify the integrand before integration over momenta. The simplest approach would be to expand the denominator in a power series over $a^{2} / k^{2}$ prior to integration over the Feynman parameters $x$ and $y$. However, it is easy to see that the second term of this naive expansion 
would lead to a divergence in the integration over $y$ when $y$ goes to zero. Of course, the initial integral is convergent, and this divergence is a result of an improper expansion. A safe approach would be to calculate first the integrals over the Feynman parameters, and only then to expand in large $k^{2}$. Emergence of the logarithm of $k^{2}$ in this expansion corresponds to the divergence which was encountered in attempting to make the large $k^{2}$ expansion prior to the Feynman parameter integration.

However, we would like to avoid tedious calculation of all integrals over $x$ and $y$ prior to the large momentum expansion. As we will demonstrate now this may be achieved with the help of a trick, which will significantly simplify further calculations. The idea is to use the following representation for the coefficient function in Eq. (B1)

$$
b c_{7}=\widetilde{b c_{7}}+\left(b c_{7}-\widetilde{b c_{7}}\right)
$$

where

$$
\widetilde{b c_{7}}=\left.(1-2 y)\left(b(x, y) c_{7}(x, y)\right)\right|_{y=0}
$$

The weight function $\left(b c_{7}-\widetilde{b c_{7}}\right)$ is obviously proportional to $y$, and, hence, the highmomenta integrals with such weight function admit naive expansion over $1 / k^{2}$ prior to integration over the Feynman parameters. Integration over $y$ in the integral with the weight function $\widetilde{b c_{7}}$ is simplified due to the presence of the factor $1-2 y=[y(1-2 y)]^{\prime}$.

Let us consider first the integral with the weight function $\widehat{b c_{7}}$

$$
\widetilde{F}(\mu)=2 \int_{0}^{1} d x \int_{0}^{x} d y \frac{(1-x)^{2}}{x}(1-2 y) \int_{0}^{\infty} d k^{2} \frac{k^{2}}{\left(k^{2}+a^{2}\right)^{2}} \Phi_{1}^{\mu}(k) .
$$

In order to calculate the high-momenta contribution we first integrate over variable $y$, expand the result in the series over $1 / k^{2}$, and then integrate over $x$

$$
\widetilde{F}^{>}(\mu)=2 \int_{\sigma}^{\infty} d k^{2}\left\{\frac{1}{4 k^{2}}-\frac{1}{3 k^{4}} \ln k\right\} \Phi_{1}^{\mu}(k)
$$

Note that we took into account only the first two terms in the expansion over $1 / k^{2}$. As we will see below, due to the special choice of the auxiliary parameter $\sigma$, these terms are sufficient for calculation of the integral above with accuracy linear in $\mu$. The remaining momentum integration is easily performed with the help of the standard integrals $V_{k m n}$ which are calculated in Appendix Q, and we obtain

$$
\begin{gathered}
\widetilde{F}^{>}(\mu)=2\left(\frac{1}{4} V_{101}-\frac{1}{3} V_{211}\right) \approx 2\left[-\frac{1}{4} \ln (2 \mu)-\frac{1}{8}-\frac{1}{4} \ln \sigma\right. \\
\left.+\frac{\mu \sigma}{2}-\frac{(\mu \sigma)^{2}}{4}-\frac{1}{\sigma^{2}}\left(\frac{1}{6} \ln \sigma+\frac{1}{12}\right)\right] .
\end{gathered}
$$

The result above is a power series in the small parameters $(\mu \sigma)$ and $1 / \sigma^{2}$. In order to get rid of higher terms in the expansion we further specify the magnitude of the auxiliary parameter $\sigma \simeq 1 / \sqrt{\mu}$. With such a choice of this parameter we need only explicitly written terms in the relationship above in order to obtain the value of the integrand with linear 
accuracy in $\mu$. The same specification of this parameter also justifies consideration of only the two first terms in the expansion over $1 / k^{2}$ above.

Calculating the low momentum integral we first expand function $\Phi_{1}^{\mu}(k)$ in the small parameter $\mu k$ (up to and including the term $(\mu k)^{2} \leq(\mu \sigma)^{2}$ )

$$
\Phi_{1}^{\mu}(k) \approx \frac{1}{2}-\mu k+(\mu k)^{2}+O\left((\mu k)^{3}\right)
$$

and then perform the momentum integration

$$
\begin{gathered}
\widetilde{F}^{<}(\mu) \approx 2 \int_{0}^{1} d x \int_{0}^{x} d y \frac{(1-x)^{2}}{x}(1-2 y)\left\{\frac{1}{2}\left[\ln \frac{\sigma^{2}+a^{2}}{a^{2}}-\frac{\sigma^{2}}{\sigma^{2}+a^{2}}\right]\right. \\
\left.-\mu\left[2 \sigma+\frac{\sigma a^{2}}{\sigma^{2}+a^{2}}-3 a \arctan \frac{\sigma}{a}\right]+\mu^{2}\left[\sigma^{2}-\frac{\sigma^{2} a^{2}}{\sigma^{2}+a^{2}}-2 a^{2} \ln \frac{\sigma^{2}+a^{2}}{a^{2}}\right]\right\} .
\end{gathered}
$$

Calculating remaining integrals and omitting the higher order terms with the help of the condition $\sigma \simeq 1 / \sqrt{\mu}$ we obtain

$$
\tilde{F}^{<}(\mu) \approx 2\left[-\frac{1}{48}+\frac{1}{4} \ln \sigma+\frac{15 \pi^{2}}{128} \mu-\frac{\mu \sigma}{2}+\frac{(\mu \sigma)^{2}}{4}+\frac{1}{\sigma^{2}}\left(\frac{1}{6} \ln \sigma+\frac{1}{12}\right)\right] .
$$

All terms with the auxiliary parameter $\sigma$ cancel in the sum of the high- and lowmomentum integrals and we obtain with linear accuracy in the small parameter $\mu$

$$
\widetilde{F}(\mu) \approx\left[-\frac{1}{2} \ln (2 \mu)-\frac{7}{24}\right]+\frac{15 \pi^{2}}{64} \mu
$$

The first term in this relationship gives the contribution of the first order in the small mass ratio to hyperfine splitting, and is of no interest to us. The second term leads to the correction of order $(m / M)^{2}$ and calculation of such terms is the main goal of this work.

Let us consider further the origin of this term. First, it is not connected with the highmomentum integral, which generated only corrections of the previous order in the mass ratio. The term which generates the correction under consideration in the low-momentum integral in Eq. (B9) and Eq. (B10) may be easily identified. This is the term which explicitly contains factor $\mu$ generated by the low-momentum expansion (see Eq. (BB8)) of the function $\Phi_{1}^{\mu}(k)$, and moreover it is the only term of order $\mu$ in the integral which remains finite when $\sigma$ goes to infinity. We may be even more concrete. It is easy to see that the only term which remains finite when the upper limit in the integral $\int_{0}^{\sigma} d k^{2} k^{2} /\left(k^{2}+a^{2}\right)^{n} \mu k$ goes to infinity has the characteristic form of $\arctan (\sigma / a)$.

Now we are ready to describe a recipe for obtaining the term of order $\mu$ in the integral in Eq. (B5) almost without calculations. First, we have to calculate only the low-momentum contribution to the integral in Eq. (B5), and consider in the integrand only the term linear in $\mu$ generated by the respective term in the low-momentum expansion of the function $\Phi_{1}^{\mu}(k)$. At the second step we calculate the momentum integral and preserve in the result only contribution of the arctangent which gives factor $\pi / 2$. At the last step we have to calculate 
the remaining double integral over the Feynman parameters. It is easy to check that this recipe immediately reproduces the term linear in $\mu$ in Eq. (B11).

Up to now we have calculated only the linear in $\mu$ contribution to the integral $\widetilde{F}(\mu)$ in Eq. (B5). However, it is easy to see that the recipe for extracting linear in $\mu$ contributions which we have just formulated is equally applicable for the total integral $F(\mu)$. The only difference between the integrals with the weight functions $b c_{7}$ and $\widetilde{b c_{7}}$ is in the details of the calculation of the high momentum contribution to the integral. The representation of the weight function in the form of the sum in Eq. (B3), simplified the calculation of the highmomentum contribution generated by the weight function $b c_{7}-\widetilde{b c_{7}}$, but as we have just seen the high-momentum contribution does not generate linear in $\mu$ contributions to the integral $F(\mu)$. Hence, we may apply the recipe above for calculation of the total contribution of order $\mu$ to the integral $F(\mu)$, the only difference being that the role of the weight function will play now the total weight function $b c_{7}$.

It is clear that the same recipe will work each time when the $\mu$-integral for the contribution to the hyperfine splitting contains function $\Phi_{1}^{\mu}(k)$ in the integrand. Moreover, one may check explicitly that the recipe is valid even when the $\mu$-integral depends on other functions $\Phi_{0,2}^{\mu}(k)$, as happens for some contributions generated by the electron factor. All respective calculations in the main body of the paper are made with the help of this recipe.

\section{APPENDIX C: STANDARD HIGH-MOMENTUM INTEGRALS}

As was mentioned in the main text and Appendix B all high-momentum contributions to hyperfine splitting may written in terms of the standard integrals

$$
V_{l m n}=\int_{\sigma}^{\infty} \frac{d k^{2}}{\left(k^{2}\right)^{l}}(\ln k)^{m} \Phi_{n}^{\mu}(k),
$$

where $l=1,2, m=0,1$ and $n=1,2$.

These integrals should be calculated with linear accuracy in $\mu$. As was discussed in Appendix $\mathrm{B}$ this means that integrals should be calculated up to and including small terms of order $(\mu \sigma)^{2}$ (we remind the reader that $\sigma \approx 1 / \sqrt{\mu}$ ).

Consider in more detail the integrals with $n=1$

$$
V_{l m 1}=\int_{\sigma}^{\infty} \frac{d k^{2}}{\left(k^{2}\right)^{l}}(\ln k)^{m}\left[-\mu k \sqrt{1+\mu^{2} k^{2}}+\mu^{2} k^{2}+\frac{1}{2}\right] .
$$

First, we change the integration variable

$$
t=\sqrt{1+\mu^{2} k^{2}}-\mu k .
$$

In terms of this new variable typical integrals acquire the form

$$
\begin{gathered}
V_{101}=\int_{0}^{t_{\sigma}} d t \frac{t\left(1+t^{2}\right)}{1-t^{2}} \\
V_{111}=\int_{0}^{t_{\sigma}} d t \frac{t\left(1+t^{2}\right)}{1-t^{2}} \ln \frac{1-t^{2}}{2 \mu t},
\end{gathered}
$$




$$
\begin{gathered}
V_{201}=4 \mu^{2} \int_{0}^{t_{\sigma}} d t \frac{t^{3}\left(1+t^{2}\right)}{\left(1-t^{2}\right)^{3}} \\
V_{211}=4 \mu^{2} \int_{0}^{t_{\sigma}} d t \frac{t^{3}\left(1+t^{2}\right)}{\left(1-t^{2}\right)^{3}} \ln \frac{1-t^{2}}{2 \mu t},
\end{gathered}
$$

where $t_{\sigma}=\sqrt{1+\mu^{2} \sigma^{2}}-\mu \sigma \approx 1-\mu \sigma+\left(\mu^{2} \sigma^{2}\right) / 2$.

Let us, for example, calculate the integral $V_{111}$

$$
V_{111}=\int_{0}^{t_{0}} d t t\left(\frac{2}{1-t^{2}}-1\right) \ln \frac{1-t^{2}}{2 \mu t} \equiv V^{\prime}+V^{\prime \prime} .
$$

The first term in Eq. (C8) may be written in the form

$$
\begin{gathered}
V^{\prime}=-\int_{0}^{t_{\sigma}} d \ln \left(1-t^{2}\right) \ln \frac{1-t^{2}}{2 \mu t} \\
=-\frac{1}{2} \ln ^{2}\left(1-t_{\sigma}^{2}\right)+\ln \left(2 \mu t_{\sigma}\right) \ln \left(1-t_{\sigma}^{2}\right)-\int_{0}^{t_{\sigma}} \frac{d t}{t} \ln \left(1-t^{2}\right) .
\end{gathered}
$$

With the help of an exact relationship

$$
1-t_{\sigma}^{2}=2 \mu \sigma t_{\sigma}
$$

$V^{\prime}$ may be written in the form

$$
\begin{gathered}
V^{\prime}=\frac{1}{2} \ln ^{2}(2 \mu)-\frac{1}{2} \ln ^{2} \sigma+\frac{1}{2} \ln ^{2} t_{\sigma}+\ln (2 \mu) \ln t_{\sigma} \\
-\int_{0}^{1} \frac{d t}{t} \ln \left(1-t^{2}\right)+\int_{t_{\sigma}}^{1} \frac{d t}{t} \ln \left(1-t^{2}\right) .
\end{gathered}
$$

Expansion over $(\mu \sigma)$ is facilitated by the relationship

$$
\ln t_{\sigma} \approx-\mu \sigma+O\left[(\mu \sigma)^{3}\right]
$$

and we obtain

$$
V^{\prime} \approx \frac{1}{2} \ln ^{2}(2 \mu)-\frac{1}{2} \ln ^{2} \sigma+\frac{\pi^{2}}{12}+(\mu \sigma)(\ln \sigma-1)+O\left[(\mu \sigma)^{3}\right] .
$$

Approximate expression for the integral $V^{\prime \prime}$ may be obtained in the same way

$$
V^{\prime \prime} \approx \frac{1}{4}+\frac{1}{2} \ln (2 \mu)+(\mu \sigma)(\ln \sigma-1)+(\mu \sigma)^{2}\left(-\ln \sigma+\frac{1}{2}\right),
$$

and then we obtain an approximation for the integral in Eq. (C8)

$$
\begin{aligned}
V_{111} & \simeq \frac{1}{2} \ln ^{2}(2 \mu)+\frac{1}{2} \ln (2 \mu)+\frac{\pi^{2}}{12}+\frac{1}{4}-\frac{1}{2} \ln ^{2} \sigma \\
& +(\mu \sigma)(2 \ln \sigma-2)+(\mu \sigma)^{2}\left(-\ln \sigma+\frac{1}{2}\right) .
\end{aligned}
$$

Approximate expressions for other integrals $V_{l m n}$ may be obtained in the same way, and they are collected in Table [II. 


\section{REFERENCES}

[1] F. G. Mariam, W. Beer, P.R. Bolton et al, Phys. Rev. Lett. 49, 993 (1982).

[2] V. W. Hughes, Z. Phys. C 56, S35 (1992).

[3] M. I. Eides, S. G. Karshenboim, and V. A. Shelyuto, Phys. Lett. 229B, 285 (1989); Pis'ma Zh. Eksp. Teor. Fiz. 50, 3 (1989) [Sov. Phys. JETP Lett. 50, 1 (1989)]; Yad. Fiz. 50, 1636 (1989) [Sov. J. Nucl. Phys.50, 1015 (1989)].

[4] M. I. Eides, S. G. Karshenboim, and V. A. Shelyuto, Phys. Lett. 249B, 519 (1990); Pis'ma Zh. Eksp. Teor. Fiz. 52, 937 (1990) [Sov. Phys. JETP Lett. 52, 317 (1990)].

[5] M. I. Eides, S. G. Karshenboim, and V. A. Shelyuto, Phys. Lett. 268B, 433 (1991); 316B, 631 (E) (1993); 319B, 545 (E) (1993); Yad. Fiz. 55,466 (1992); 57, 1343 (E) (1994) [Sov. J. Nucl. Phys. 55, 257 (1992)].

[6] M. I. Eides, S. G. Karshenboim, and V. A. Shelyuto, Phys. Lett. 312B, 358 (1993); Yad. Fiz. 57, 1309 (1994) [Phys. Atom. Nucl. 57 (1994) 1240]; Yad. Fiz. 57, 2246 (1994) [Phys. Atom. Nucl. 57, 2158 (1994)].

[7] T. Kinoshita and M. Nio, Phys. Rev. Lett. 72, 3803 (1994).

[8] M. I. Eides and V. A. Shelyuto, Pis'ma Zh. Eksp. Teor. Fiz. 61, 465 (1995) [JETP Letters 61, 478 (1995)]; Phys. Rev. A 52, 954 (1995).

[9] T. Kinoshita and M. Nio, Phys. Rev. D 53, 4909 (1996).

[10] S. Karshenboim, Zh. Eksp. Teor. Fiz. 103, 1105 (1993) [Sov. Phys. JETP 76, 541 (1993)]; Z. Phys. D 36, 11 (1996).

[11] P. Lepage, unpublished, as cited in [7].

[12] S. A. Blundell, K. T. Cheng, and J. Sapirstein, Phys. Rev. Lett. 78, 4914 (1997).

[13] M. I. Eides and V. A. Shelyuto, Phys. Lett. 146B, 241 (1984).

[14] M. I. Eides, S. G. Karshenboim, and V. A. Shelyuto, Phys. Lett. 216B, 405 (1989).

[15] K. Pachucki, Phys. Rev. A 54, 1994 (1996).

[16] T. Kinoshita and M. Nio, Phys. Rev. D 55, 7267 (1997).

[17] J. R. Sapirstein, E. A. Terray, and D. R. Yennie, Phys. Rev. Lett. 51, 982 (1983); Phys. Rev. D 29, 2290 (1984).

[18] M. I. Eides, S. G. Karshenboim, and V. A. Shelyuto, Phys. Lett. 177B, 425 (1986); Yad. Fiz. 44, 1118 (1986) [Sov. J. Nucl. Phys. 44, 723 (1986)]; Zh. Eksp. Teor. Fiz. 92, 1188 (1987) [Sov. Phys. JETP 65, 664 (1987)]; Yad. Fiz. 48, 1039 (1988) [Sov. J. Nucl. Phys. 48, 661 (1988)].

[19] M. I. Eides, V. Yu. Brook, S. G. Karshenboim, and V. A. Shelyuto, Phys. Lett. 216B, 401 (1989).

[20] M. I. Eides, S. G. Karshenboim, and V. A. Shelyuto, Ann. Phys. (NY) 205, 231 (1991).

[21] N. Kroll and F. Pollock, Phys. Rev. 84, 594 (1951); 86, 876 (1952).

[22] R. Karplus, A. Klein, and J. Schwinger, Phys. Rev. 84, 597 (1951); R. Karplus and A. Klein, ibid. 85, 972 (1952).

[23] W. E. Caswell and G. P. Lepage, Phys. Rev. Lett. 41, 1092 (1978).

[24] E. A. Terray and D. R. Yennie, Phys. Rev. Lett. 48, 1803 (1982).

[25] M. I. Eides, S. G. Karshenboim, and V. A. Shelyuto, Phys. Lett. 202B, 572 (1988); Zh. Eksp. Teor. Fiz. 94, 42 (1988) [Sov.Phys. JETP 67, 671 (1988)].

[26] M. I. Eides, S. G. Karshenboim, and V. A. Shelyuto, Ann. Phys. (NY) 205, 291 (1991). 


\section{TABLES}

TABLE I. Coefficients in the Electron-Line Factor

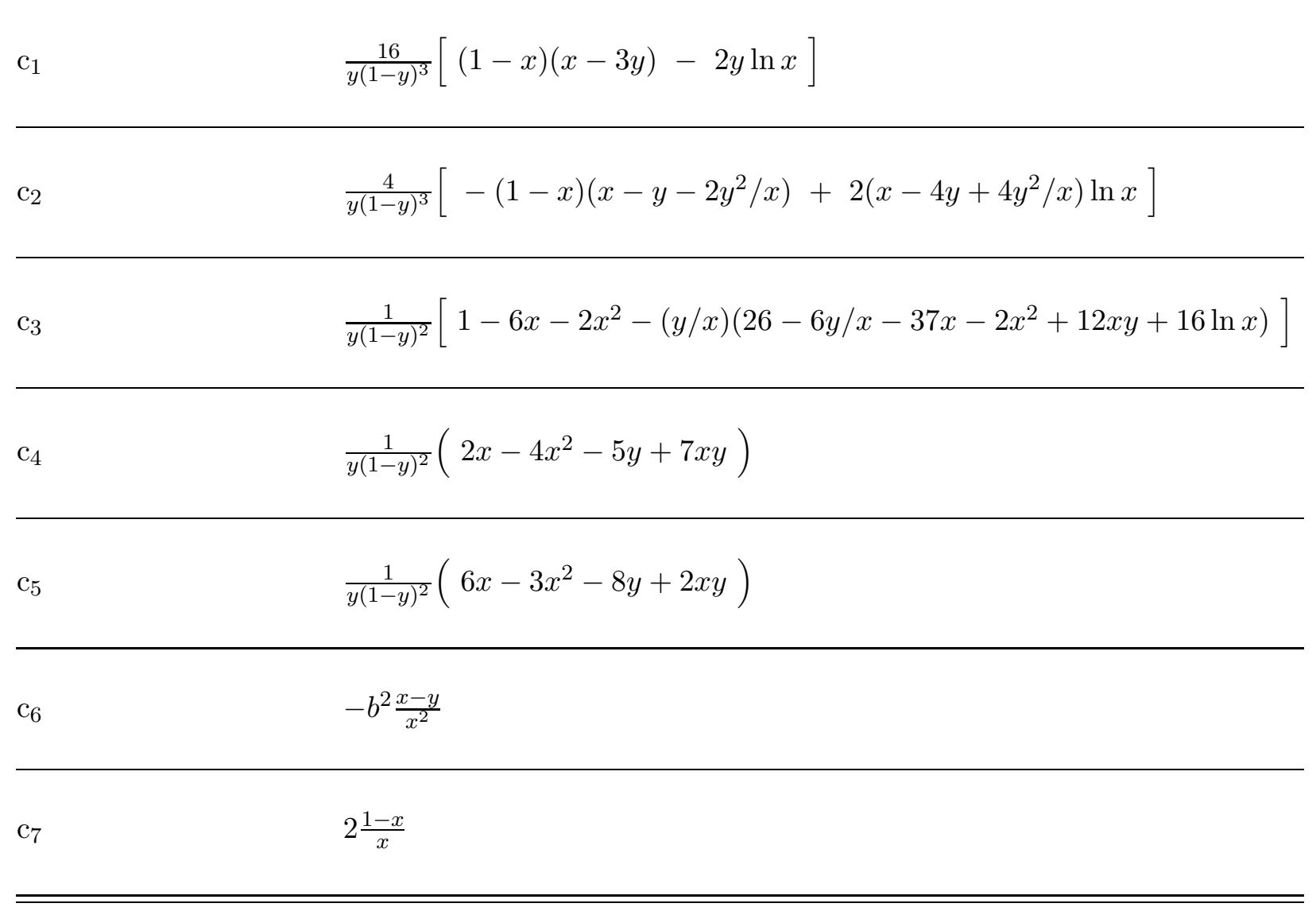


TABLE II. Electron-Factor Contributions to Hyperfine Splitting

\begin{tabular}{|c|c|}
\hline$\delta E_{i}$ & $\alpha(Z \alpha)\left(\frac{m}{M}\right)^{2} E_{F}$ \\
\hline$\delta E_{123}$ & $\left(-6 \ln 2-\frac{39}{32}\right)$ \\
\hline$\delta E_{4}$ & $\frac{3}{16}$ \\
\hline$\delta E_{5}$ & $-\frac{3}{32}$ \\
\hline$\delta E_{6}$ & $-\frac{3}{32}$ \\
\hline$\delta E_{7}$ & $-\frac{9}{32}$ \\
\hline$\delta E_{\mathrm{el}}=\sum_{i=1}^{7} \delta E_{i}$ & $\left(-6 \ln 2-\frac{3}{2}\right)$ \\
\hline
\end{tabular}


TABLE III. Approximate expressions for the integrals $V_{l m n}$

\begin{tabular}{|c|c|}
\hline$V_{100}$ & $2 \ln (2 \mu)-2+2 \ln \sigma-(\mu \sigma)$ \\
\hline$V_{110}$ & $-\ln ^{2}(2 \mu)+2 \ln (2 \mu)-\frac{\pi^{2}}{6}-2+\ln ^{2} \sigma+(\mu \sigma)(-\ln \sigma+1)$ \\
\hline$V_{101}$ & $-\ln (2 \mu)-\frac{1}{2}-\ln \sigma+2(\mu \sigma)-(\mu \sigma)^{2}$ \\
\hline$V_{111}$ & $\frac{1}{2} \ln ^{2}(2 \mu)+\frac{1}{2} \ln (2 \mu)+\frac{\pi^{2}}{12}+\frac{1}{4}-\frac{1}{2} \ln ^{2} \sigma+(\mu \sigma)(2 \ln \sigma-2)+(\mu \sigma)^{2}\left(-\ln \sigma+\frac{1}{2}\right)$ \\
\hline$V_{200}$ & $-\frac{1}{\sigma^{2}}$ \\
\hline$V_{201}$ & $\frac{1}{2 \sigma^{2}}$ \\
\hline$V_{202}$ & $\frac{1}{8 \sigma^{2}}$ \\
\hline$V_{210}$ & $-\frac{1}{\sigma^{2}} \cdot\left(\ln \sigma+\frac{1}{2}\right)$ \\
\hline$V_{211}$ & $\frac{1}{2 \sigma^{2}} \cdot\left(\ln \sigma+\frac{1}{2}\right)$ \\
\hline$V_{212}$ & $\frac{1}{8 \sigma^{2}} \cdot\left(\ln \sigma+\frac{1}{2}\right)$ \\
\hline
\end{tabular}

